\title{
Mass Transfer and Heat Generation Effects on MHD Free Convection Flow past an Inclined Vertical Surface in a Porous Medium
}

\author{
M.G. Reddy ${ }^{1 \dagger}$ and N.B. Reddy ${ }^{2}$ \\ ${ }^{1}$ Deparment of Mathematics, Acharya Nagarjuna University Ongole Campus, Ongole - 523 001, India \\ ${ }^{2}$ Department of Mathematics, Sri Venkateswara University, Tirupati- 517 502, India \\ $\dagger$ Corresponding Author Email: drmgranu@gmail.com
}

(Received August 18, 2008; accepted May 5, 2010)

\begin{abstract}
A steady two-dimensional MHD free convection and mass transfer flow past an inclined semi-infinite vertical surface in the presence of heat generation and a porous medium has been studied numerically. The governing partial differential equations are reduced to a system of ordinary differential equations by introducing similarity transformations. The non-linear similarity equations are solved numerically by applying the Runge-Kutta method of fourth order with shooting technique. The numerical results are presented graphically for different values of the parameters. Finally, the numerical values of the local skin-friction coefficient, local Nusselt number and Sherwood number are shown in Table 1.
\end{abstract}

Keywords: MHD, Heat generation, Mass transfer, Inclined vertical surface.

\section{NOMENCLATURE}

$\begin{array}{ll}B_{0} & \text { Applied magnetic field } \\ C & \text { Concentration } \\ c_{p} & \text { Specific heat at constant pressure } \\ D & \text { Mass diffusivity } \\ f & \text { Dimensionless stream function } \\ g & \text { Acceleration due to gravity } \\ G r & \text { Local temperature Grashof number } \\ G m & \text { Local mass Grashof number } \\ K & \text { Permeability parameter } \\ k & \text { Thermal conductivity of the fluid } \\ M & \text { Magnetic field parameter } \\ N u & \text { Nusselt number } \\ \operatorname{Pr} & \text { Prandtl number } \\ Q_{0} & \text { Heat generation constant } \\ Q & \text { Heat generation parameter }\end{array}$

\section{INTRODUCTION}

The problem of free convection and mass transfer flow of an electrically conducting fluid past an inclined vertical surface under the influence of a magnetic field

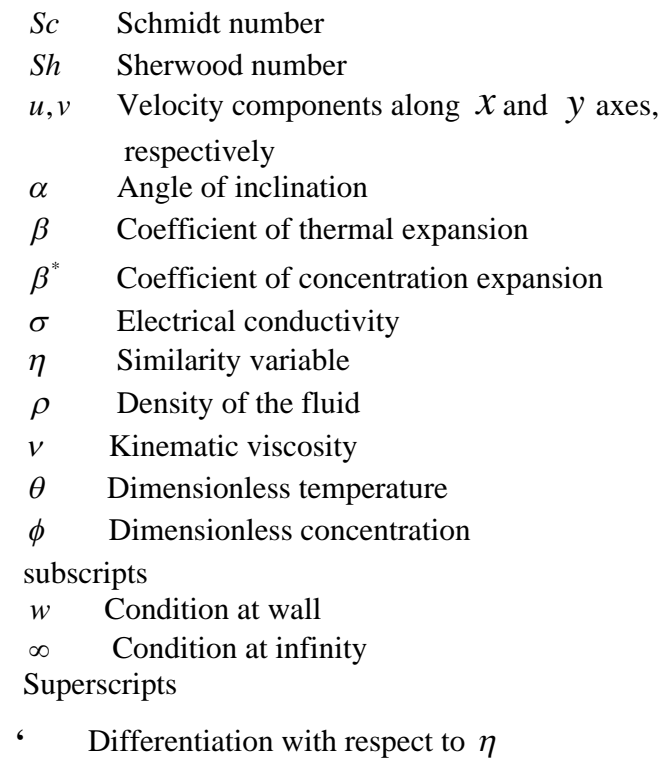

- $\quad$ Differentiation with respect to $\eta$

has attracted interest in view of its application to geophysics, astrophysics and many engineering problems, such as cooling of nuclear reactors, the boundary layer control in aerodynamics. Hossain et al. (1996) studied the free convection flow from an 
isothermal plate inclined at a small angle to the horizontal. Recently, Anghel et al. (2001) presented a numerical solution of free convection flow past an inclined surface. Chen (2004) performed an analysis to study the natural convection flow over a permeable inclined surface with variable wall temperature and concentration.

The study of the heat generation or absorption in moving fluids is important problems dealing with chemical reactions and those concerned with dissociating fluids. Vajravelu and Hadjinicolaou (1993) studied the heat transfer boundary layer of a viscous fluid over a stretching sheet with internal heat generation. Hossain et al. (2004) studied the problem of natural convection flow along a vertical wavy surface in the presence of heat generation/absorpation.

The aim of the present paper is to study mass transfer and heat generation effects on MHD free convection flow past an inclined vertical plate in a porous medium. The dimensionless equations are solved numerically. The effects of various physical parameters on the velocity, temperature and concentration are shown graphically.

\section{MATHEMATICAL ANALYSIS}

Consider a steady two hydromagnetic flow of a viscous incompressible, electrically conducting fluid past a semi-infinite inclined porous plate with an acute angle $\alpha$ to the vertical. The flow is assumed to be in the $x$ direction, which is taken along the semi-infinite inclined porous plate and $y$ - axis normal to it. A magnetic field of uniform strength $B_{0}$ is introduced normal to the direction of the flow. In the analysis, we assume that the magnetic Reynolds number is much less than unity so that the induced magnetic field is neglected in comparison to the applied magnetic field. It is also assumed that all fluid properties are constant except that of the influence of the density variation with temperature and concentration in the body force term. The surface is maintained at a constant temperature $T_{w}$, which is higher than the constant temperature $T_{\infty}$ of the surrounding fluid and the concentration $C_{w}$ is greater than the constant concentration $C_{\infty}$. Then, under the usual Boussinesq's and boundary layer approximations, the governing equations are Continuity equation

$$
\frac{\partial u}{\partial x}+\frac{\partial v}{\partial y}=0
$$

Momentum equation

$$
\begin{gathered}
u \frac{\partial u}{\partial x}+v \frac{\partial u}{\partial y}=v \frac{\partial^{2} u}{\partial y^{2}}+g \beta\left(T-T_{\infty}\right) \cos \alpha \\
+g \beta^{*}\left(C-C_{\infty}\right) \cos \alpha-\frac{\sigma B_{0}^{2}}{\rho} u-\frac{v}{K^{\prime}} u
\end{gathered}
$$

Energy equation $u \frac{\partial T}{\partial x}+v \frac{\partial T}{\partial y}=\frac{k}{\rho c_{p}} \frac{\partial^{2} T}{\partial y^{2}}+\frac{Q_{0}}{\rho c_{p}}\left(T-T_{\infty}\right)$

Species equation

$u \frac{\partial C}{\partial x}+v \frac{\partial C}{\partial y}=D \frac{\partial^{2} C}{\partial y^{2}}$

The boundary conditions for the velocity, temperature and concentration fields are

$u=0, v=0, T=T_{w}, C=C_{w}$ at $y=0$

$u \rightarrow 0, T \rightarrow T_{\infty}, C \rightarrow C_{\infty}$ as $y \rightarrow \infty$

In order to obtain a similarity solution of the problem we introduce the following non-dimensional variables

$$
\begin{aligned}
& \eta=y \sqrt{\frac{U_{\infty}}{v x}}, \quad \theta(\eta)=\frac{T-T_{\infty}}{T_{w}-T_{\infty}}, \\
& u=U_{\infty} f^{\prime}(\eta), G r=\frac{g \beta\left(T_{w}-T_{\infty}\right) x}{U_{\infty}^{2}}, \\
& G m=\frac{g \beta^{*}\left(C_{w}-C_{\infty}\right) x}{U_{\infty}^{2}}, \phi(\eta)=\frac{C-C_{\infty}}{C_{w}-C_{\infty}} \\
& v=\frac{1}{2} \sqrt{\frac{U_{\infty} v}{x}}\left(\eta f^{\prime}-f\right), \operatorname{Pr}=\frac{v \rho c_{p}}{k}, \\
& M=\frac{\sigma B_{0}^{2} x}{\rho U_{\infty}}, K=\frac{v x}{K^{\prime} U_{\infty}}, \\
& Q=\frac{Q_{0} x}{\rho c_{p} U_{\infty}}, S c=\frac{v}{D}
\end{aligned}
$$

Now substituting Eq. (6) in Eqs. (2) - (4), we obtain

$$
\begin{aligned}
& f^{\prime \prime \prime}+\frac{1}{2} f f^{\prime \prime}+G r \theta \cos \alpha \\
& +G m \phi \cos \alpha-(M+K) f^{\prime}=0 \\
& \theta^{\prime \prime}+\frac{1}{2} \operatorname{Pr} f \theta^{\prime}+\operatorname{Pr} Q \theta=0 \\
& \phi^{\prime \prime}+\frac{1}{2} S c f \phi^{\prime}=0
\end{aligned}
$$

The corresponding boundary conditions are

$$
\begin{aligned}
& f=0, f^{\prime}=0, \theta=1, \phi=1 \text { at } \eta=0 \\
& f^{\prime} \rightarrow 0, \theta \rightarrow 0, \phi \rightarrow 0 \text { as } \eta \rightarrow \infty
\end{aligned}
$$

The local skin-friction, Nusselt number and Sherwood number are important physical parameters for this type of boundary layer flow.

The local skin-friction in non-dimensional form is

$$
C_{f}=2(\mathrm{Re})^{-\frac{1}{2}} f^{\prime \prime}(0)
$$

The local Nusselt number in non-dimensional form is

$$
N u=-(\operatorname{Re})^{\frac{1}{2}} \theta^{\prime}(0)
$$


The local Sherwood number in non-dimensional form is

$$
S h=-(\operatorname{Re})^{\frac{1}{2}} \phi^{\prime}(0)
$$

where $\operatorname{Re}=\frac{U_{\infty} x}{v}$ is the Reynolds number

\section{NUMERICAL SOLUTION AND DISCUSSION}

The Eqs. (7) - (9) with boundary conditions (10) were solved numerically using the Runge-Kutta fourth order with Shooting method. As a result of the numerical calculations, the dimensionless velocity, temperature and concentration distributions for the flow are obtain $\mathrm{Pr}=0.71$ ed from the Eqs. (7) - (9) and are displayed in Figs. 1-7 for different values of $G r, G m, M, K, \alpha$, $Q$ and $S c$. The value of the Prandtl number is chosen for air (i.e. $\operatorname{Pr}=0.71$ ).

In order to ascertain the accuracy of the numerical results, the present study is compared with the previous study. The velocity profiles for $G r=2.0, G m=2.0$, $K=0.5, \operatorname{Pr}=0.71, Q=0.0, S c=0.6, \alpha=30^{\circ}$ are compared with the available solution of Sivasankaran et al. (2006) in Fig. 1. It is observed that the present results are in good agreement with that of Sivasankaran et al. (2006)

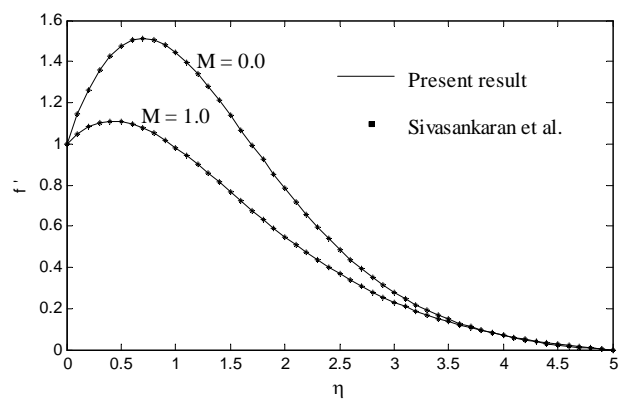

Fig.1. Comparison of velocity profiles

For various values of thermal Grashof number and solutal Grashof number, the velocity profiles are plotted in Fig.2. The thermal Grashof number $G r$ signifies the relative effect of the thermal buoyancy force to the viscous hydrodynamic force in the boundary layer. As expected, it is observed that there is a rise in the velocity due to the enhancement of thermal buoyancy force. Here, the positive values of $G r$ correspond to cooling of the surface. Also, as Gr increases, the peak values of the velocity increases rapidly near the porous plate and then decays smoothly to the free stream velocity. The solutal Grashof number $G m$ defines the ratio of the species buoyancy force to the viscous hydrodynamic force. As expected, the fluid velocity increases and the peak value is more distinctive due to increase in the species buoyancy force. The velocity distribution attains a distinctive maximum value in the vicinity of the plate and then decreases properly to approach the free stream value. It is noticed that the velocity increases with increasing values of the solutal Grashof number.

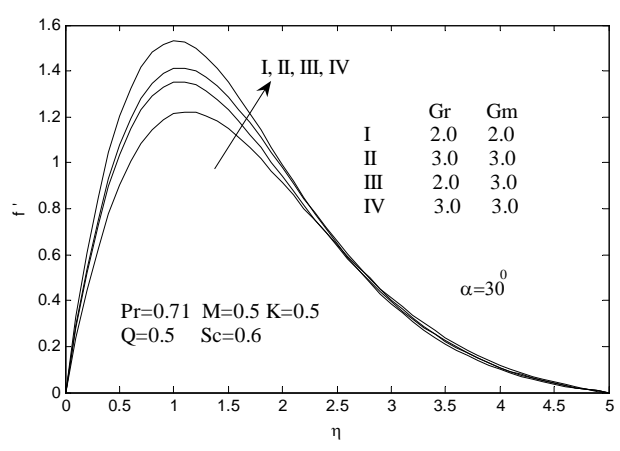

Fig.2. Effect of $G r$ and $G m$ on velocity profiles

The velocity profiles are shown in Fig. 3 for different values of $M$ and $K$. The velocity decreases with an increase in the magnetic parameter. It is because that the application of transverse magnetic field will result a resistive type force (Lorentz force) similar to drag force which tends to resist the fluid flow and thus reducing its velocity. Also, the boundary layer thickness decreases with an increase in the magnetic parameter. The parameter $K$ as defined in Eq. (6) is inversely proportional to the actual permeability $K^{\prime}$ of the porous medium. An increase in $K$ will therefore increase the resistance of the porous medium (as the permeability physically becomes less with increasing $K^{\prime}$ ) which will tend to decelerate the flow and reduce the velocity. This behaviour is evident from Fig.3.

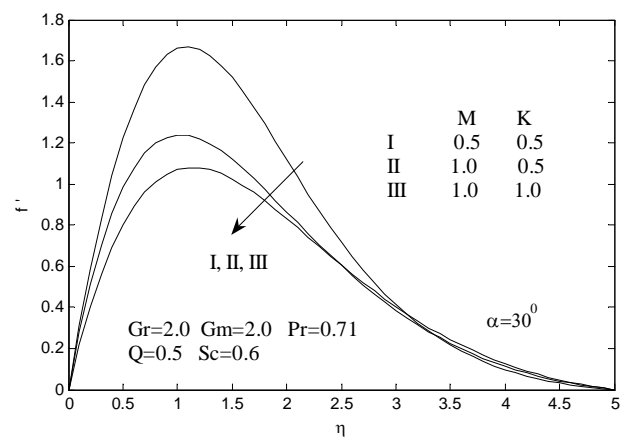

Fig.3. Effect of $M$ and $K$ on velocity profiles

The effect of inclination parameter $\alpha$ on the velocity are shown in Fig.4. It is observed that the fluid (air) velocity is decreased for increasing angle $\alpha$.

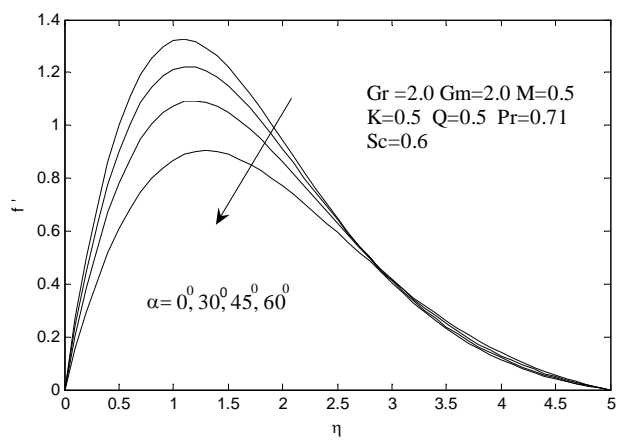

Fig.4. Effect of $\alpha$ on velocity profiles 
Figure 5 shows the effect of the heat generation parameter $Q$ on the velocity distribution. It is seen from this figure that when heat is generated the buoyancy force increases, which induces the flow rate to increase, giving rise to the increase in the velocity profiles. The effect of $Q$ on temperature profiles are shown in Fig.6. It is clear that the heat generation increases, the temperature is also increases.

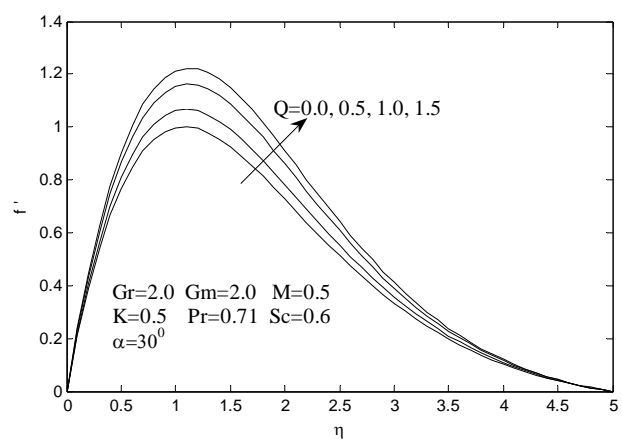

Fig.5. Effect of $Q$ on velocity profiles

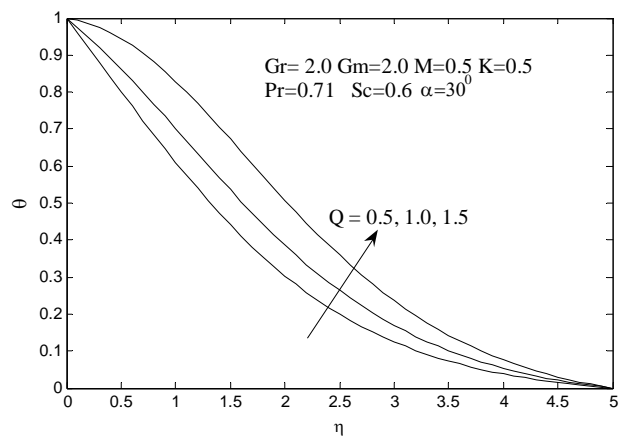

Fig.6. Effect of $Q$ on temperature profiles

The influence of thermal Schmidt number $S c$ on the velocity is shown in Fig. 7. The Schmidt number embodies the ratio of the momentum to the mass diffusivity. The Schmidt number therefore quantifies the relative effectiveness of momentum and mass transport by diffusion in the hydrodynamic (velocity) and concentration (species) boundary layers. As the Schmidt number increases the concentration decreases. This causes the concentration buoyancy effects to decrease yielding a reduction in the fluid velocity. It is observed that an increase in Sc, the velocity decreases.

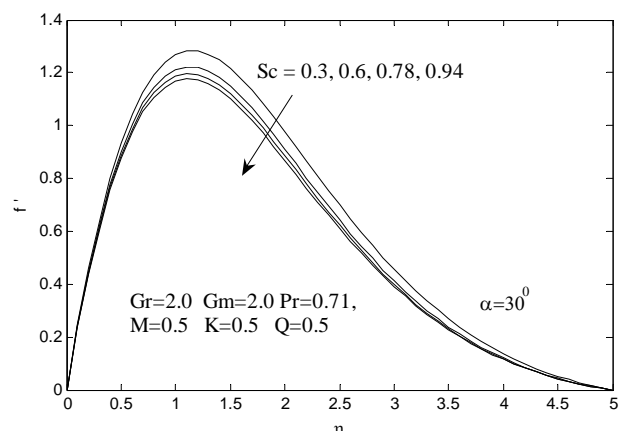

Fig.7. Effect of $S c$ on velocity profiles
The effect of the Schmidt number Sc on concentration profiles are shown in Fig. 8. A rise in Sc strongly suppresses concentration levels in the boundary layer regime. All profiles decay monotonically from the surface (wall) to the free stream. Sc embodies the ratio of momentum diffusivity $(v)$ to molecular diffusivity

$(D)$. It is conclude that, an increase in Sc, the concentration decreases.

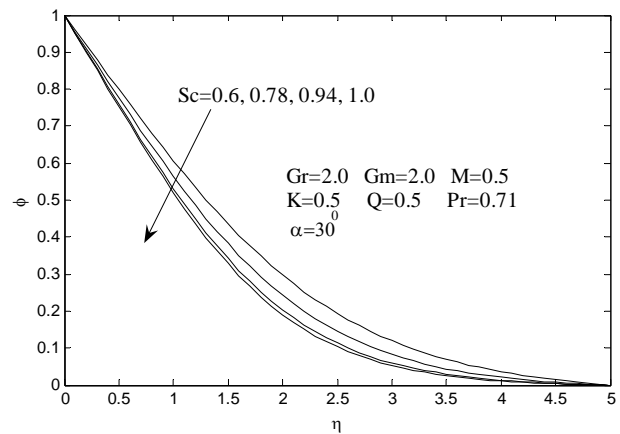

Fig.8. Effect of Sc on concentration profiles

The effect of various physical parameters for the local skin-frication coefficient $C_{f}$, the local Nusselt number $N u$ and the Sherwood number $S h$ are shown in Table 1. ( $\operatorname{Pr}=0.71$ and $\alpha=30^{\circ}$ ). It is observed that an increase in $G r$ or $G m$, the local skin-friction coefficient, Nusselt number, Sherwood number are increases. The heat generation increase, the skinfriction coefficient, Sherwood number increase, where as Q increase the Nusselt nuber is decrease. Also, an increase in $M$ or $K$ or $S c$, the local skin-friction coefficient, Nusselt number, Sherwood number is decreases.

\section{Conclusions}

In this paper, a mathematical model has been presented for the mass transfer and heat generation effects on MHD free convection flow past an inclined vertical plate in a porous medium. Using the similarity transformations a set of ordinary differential equations has been derived for the conservation of mass, momentum and species diffusion in the boundary layer. These nonlinear, coupled differential equations have been solved under physically valid boundary conditions using Runge-Kutta fourth order technique with shooting method. The particular conclusions drawn from the study can be listed as follows:

1. The velocity increases with the increase thermal Grashof number and solutal Grashof number.

2. The velocity decreases with an increase in the magnetic parameter. Also, the boundary layer thickness decreases with an increase in the magnetic parameter.

3. An increase in the heat generation results in increasing velocity and temperature within the boundary layer.

4. The velocity as well as concentration decreases with an increase in the Schmidt number. 
M.G. Reddy and N. B. Reddy / JAFM, Vol. 4, No. 2, Issue 1, pp. 7-11, 2011.

Table 1 Numerical values of $C_{f}, N u$ and $S h$ for $\operatorname{Pr}=0.71$ and $\alpha=30^{\circ}$

\begin{tabular}{|c|c|c|c|c|c|c|c|c|}
\hline$G r$ & $G m$ & $M$ & $K$ & $Q$ & $S c$ & $C_{f}$ & $N u$ & $S h$ \\
\hline 2.0 & 2.0 & 0.5 & 0.5 & 0.5 & 0.6 & 2.28697 & 0.08245 & 0.43260 \\
3.0 & 2.0 & 0.5 & 0.5 & 0.5 & 0.6 & 2.82219 & 0.12267 & 0.45285 \\
2.0 & 3.0 & 0.5 & 0.5 & 0.5 & 0.6 & 2.75604 & 0.11471 & 0.44892 \\
2.0 & 2.0 & 1.0 & 0.5 & 0.5 & 0.6 & 2.07847 & 0.05676 & 0.42036 \\
2.0 & 2.0 & 0.5 & 1.0 & 0.5 & 0.6 & 2.04279 & 0.05232 & 0.41826 \\
2.0 & 2.0 & 0.5 & 0.5 & 1.0 & 0.6 & 2.58995 & -0.48870 & 0.4517 \\
2.0 & 2.0 & 0.5 & 0.5 & 0.5 & 0.78 & 2.26675 & 0.07930 & 0.45990 \\
\hline
\end{tabular}

\section{REFERENCES}

Anghel, M., M.A. Hossain, and S. Zeb (2001). Combined heat and mass transfer by free

convection past an inclined flat plate. Int. J. Appl. Mech. And Engg 2, 473-497.

Chen, C.H. (2004). Heat and mass transfer in MHD flow with variable wall temperature and concentration. Acta Mechanica.172, 219-235.

Hossain, M.A., I. Pop, and M. Ahamad (1996). MHD free convection flow from an isothermal plate, J. Theo. And Appl. Mech. 1, 94207.

Hossain, M.A., M.M. Molla, and L.S. Yao (2004). Natural convection flow along a Vertical wavy surface in the presence of heat generation/absorption. Int. Therm. Sci.43, 157163.

Sivasankaran, S., P. Bhuvaneswari, P. Kandaswamy, and E. K. Ramasami (2006). Lie Group Analysis of Natural Convection Heat and Mass Transfer in an Inclined Surface. Non-linear Analysis; Modeling and Control I (1), 201-212.

Vajravelu, K. and A. Hadjinicolaou (1993). Heat transfer in a viscous fluid over a stretching with internal heat generation. Int. Comm. Heat Mass Transfer 20, 417-430. 